\title{
Émissions gazeuses à partir des vides miniers dans le bassin ferrifère lorrain
}

Z. POKRYSZKA

D. GRABOWSKI

INERIS

Parc technologique ALATA

$B P n^{\circ} 2$

60550 Verneuil-en-Halatte david.grabowski@ineris.fr zbigniew.pokryszka@ineris.fr
Une étude menée dans le bassin ferrifère lorrain a permis de mettre en évidence des émanations de mélanges gazeux sous-oxygénés et chargés en certains gaz nocifs (dioxyde de carbone, radon...), dans des agglomérations, en relation avec d'anciens travaux miniers souterrains. Les investigations effectuées in situ ont montré que les écoulements gazeux à I'origine de ces émanations sont liés au phénomène de tirage naturel. Une étude est en cours afin d'expliquer l'origine de la modification de la composition de l'air au sein des vieux travaux miniers.

Mots-clés: gaz, émanation, mine, écoulement, pyrite.

\section{Gas emissions}

from mining voids in the iron basin of Lorraine

A study carried out in the inon basin of Lorraine (France) put the spot on the existence of under-oxygenated and noxious gas emissions (carbon dioxyde, radon...) in built-up areas related to former mining works. Site investigations showed that the gas flow was mainly due to natural ventilation mechanisms. A further study is in progress in order to better understand the origin of the atmosphere modification within the old mine workings.

Key words : gas, emission, mine, flow, pyrite. 


\section{Introduction}

Certaines zones urbanisées du bassin ferrifère lorrain sont affectées par des émanations d'air vicié (mélanges gazeux sous-oxygénés et chargés en certains gaz nocifs). Ces émanations se sont manifestées de manière très spectaculaire dans l'agglomération de Moyeuvre-Grande, en Moselle (Fig, 1), et plus précisément dans certains quartiers situés à l'aplomb ou á proximité immédiate d'anciens travaux miniers souterrains. Elles se traduisaient notamment par des dysfonctionnements de gazinières et de chaudières dans les habitations.

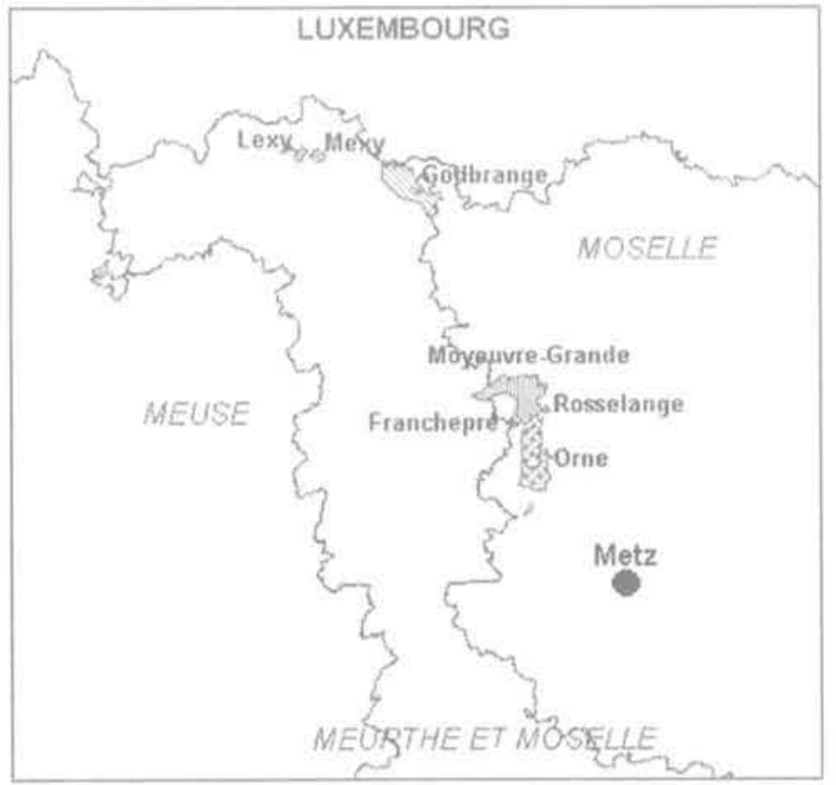

FIG. 1 Localisation des réservoirs miniers étudiés Localisation of the studied mining reservoirs.
A la demande de la Direction régionale de l'industrie, de la recherche et de l'environnement (DRIRE) Lorraine, l'Institut national de l'environnement industriel et des risques (INERIS) a entrepris, en 1999, une série d'investigations, afin de reconnaitre l'ampleur et I'étendue géographique du problème. Dans un premier temps, les investigations ont concerné le secteur du réservoir souterrain constitué par les anciens travaux miniers de Moyeuvre-Grande et partiellement les réservoirs voisins dits de Rosselange, de Franchepré et de l'Orne. Les principales caractéristiques des réservoirs étudiés sont données dans le tableau I.

Ces études ont permis de mettre en évidence des émanations d'air vicié, en relation avec les anciens travaux miniers non ou partiellement ennoyés (Pokryszka et Grabowski, 2002). Ainsi, nous avons pu observer des teneurs anormalement élevées en $\mathrm{CO}_{2}$ (jusqu'à environ $6 \%$ ) et de faibles teneurs en $\mathrm{O}_{2}$ (jusqu'a $13 \%$ ). En parallèle, les mesures effectuées par l'Institut de protection et de sûreté nucléaire (IPSN) et la Direction des affaires sanitaires et sociales (DASS) ont mis en évidence une quantité importante de radon dans les mélanges émis (jusqu'à $15000 \mathrm{~Bq} / \mathrm{m}^{3}$ ).

Dans plusieurs cas, ces émissions affectent directement les habitations. Il s'agit principalement de certaines habitations restant en contact direct avec les orifices miniers (galeries d'accès) ou avec les vieux travaux superficiels,

Dans un second temps, une campagne de mesures a été effectuée dans d'autres réservoirs miniers non ennoyés de la partie nord du bassin ferrifère : Mexy, Lexy et Godbrange (Fig. 1 et Tableau 1). Cette dernière a également permis d'identifier un phénomène d'émissions gazeuses très similaire à celui observé à Moyeuvre-Grande. Néanmoins, son intensité et son degré de modification de l'atmosphère au sein des vieux travaux miniers se sont fort heureusement avérés moindres (Pokryszka et Grabowski, 2002).

Depuis, l'INERIS poursuit des travaux de recherche destinés à comprendre le phénomène d'émissions gazeuses à partir des mines de fer. Ces travaux sont

TABLEAUII Caractéristiques des réservoirs étudiés.

Feature of the studied reservoirs (old mine workings).

\begin{tabular}{|c|c|c|c|c|c|}
\hline $\begin{array}{c}\text { Nom } \\
\text { du réservoir }\end{array}$ & $\begin{array}{c}\text { Moyeuvre-Grande } \\
\text { Orne } \\
\text { Franchepre }\end{array}$ & Rosselange & Lexy & Mexy & Godbrange \\
\hline Superficie $\left(\mathrm{Mm}^{2}\right)$ & 3,56 & 2,52 & & & \\
\hline Volumes $\left(\mathrm{Mm}^{3}\right)$ & & & 1,3 & 0,7 & $>30$ \\
\hline $\begin{array}{c}\text { Périodes } \\
\text { d'exploitation }\end{array}$ & $1873-1993$ & $1890-1935$ & $1867-1932$ & $1871-1901$ & $18 ? ?-1978$ \\
\hline $\begin{array}{c}\text { Nombre } \\
\text { d'orifices suivis }\end{array}$ & 18 & 3 & 3 & 2 & 5 \\
\hline $\begin{array}{l}\text { Cotes des orifices } \\
\text { (m NGF) }\end{array}$ & 170 à 180 & 170 à 180 & 260 à 270 & 280 à 295 & 330 à 360 \\
\hline $\begin{array}{l}\text { Cotes des vieux travaux } \\
\text { (m NGF) }\end{array}$ & 170 à 180 & 170 ả 180 & 260 à 280 & 270 à 290 & 360 à 350 \\
\hline $\begin{array}{l}\text { Cotes des plateaux } \\
(\mathrm{m} \text { NGF) }\end{array}$ & 300 à 330 & 310 à 325 & 300 à 350 & 350 à 375 & 410 à 450 \\
\hline $\begin{array}{c}\text { Différence } \\
\text { d'altitude orifice/plateau (m) }\end{array}$ & 120 à 160 & 130 à 155 & 30 à 90 & 55 à 95 & 50 à 120 \\
\hline
\end{tabular}


réalisés dans le cadre d'un programme financé par le ministère de l'Économie, des Finances et de l'Industrie, en collaboration avec la DRIRE Lorraine et GEODERIS (pôle de conseil et d'appui technique aux pouvoirs publics). Ils s'inscrivent également dans le cadre du programme de recherche du GISOS (Groupement d'intérêt scientifique et de recherche sur l'impact et la sécurité des ouvrages souterrains).

\section{2}

\section{Risques engendrés par les émissions gazeuses}

Dans une atmosphère normale, la teneur volumique en oxygène est de $20,9 \%$ et celle en dioxyde de carbone est de $0,03 \%$.

Les conséquences sur la santé humaine d'un enrichissement en $\mathrm{CO}_{2}$ et d'un appauvrissement en $\mathrm{O}_{2}$ de l'atmosphère respirée sont multiples et varient en gravité selon les teneurs de ces deux gaz. Elles vont de simples maux de têtes, pour des teneurs comprises entre 3 et $4 \%$ pour le $\mathrm{CO}_{2}$ et entre 14 et $16 \%$ pour $\mathrm{O}_{2}$, à la mort, pour des teneurs en $\mathrm{O}_{2}$ inférieures à $6 \%$ et en $\mathrm{CO}_{2}$ supérieures à $10 \%$ (Monomakhoff, 1978).

Quant au radon, il agit essentiellement sur l'épithélium pulmonaire et favorise le développement de cancer dans le cas d'une exposition prolongée à des teneurs supérieures à $500-1000 \mathrm{~Bq} / \mathrm{m}^{3}$ (Barrier et Lorentz, 2001).

Au-delà de ces effets cliniques, les dysfonctionnements constatés des appareils de combustion (chaudières, gazinières), liés à la présence d'air vicié, sont de nature à augmenter le risque d'explosion ou d'émission de CO dans les habitations affectées.

Le niveau des teneurs en gaz concernés, observé dans les secteurs étudiés du bassin ferrifère lorrain, ne présentait pas, lors des différentes campagnes des mesures, de danger immédiat. Cependant, dans certains cas, ces teneurs étaient relativement proches du niveau où les effets nuisibles peuvent se manifester par une accumulation du déficit en oxygène et des teneurs en dioxyde de carbone excessives.

Ces considérations ont amené I'INERIS à poursuivre ses investigations, afin de comprendre les mécanismes à l'origine des émanations gazeuses et de trouver des solutions appropriées à ce problème.

\section{3}

\section{Mécanisme des écoulements gazeux}

Afin de chercher une explication quant à l'origine des écoulements animant les échanges gazeux entre les vieux travaux miniers et l'atmosphère extérieure, deux stations de mesures ont été mises en place à l'entrée de deux anciennes galeries choisies dans le réservoir de Moyeuvre-Grande.

Ces stations permettent de suivre en continu l'intensité et le sens de l'écoulement d'air, la température et la teneur en certains composants du gaz émis $\left(\mathrm{O}_{2}, \mathrm{CO}_{2}\right.$ et radon), ainsi que la température extérieure et la pression barométrique.

Les figures 2 et 3 présentent les résultats les plus intéressants obtenus à partir des mesures en continu effectuées entre les mois d'avril 2000 et de décembre 2002. Compte tenu de la quantité très importante de données (une analyse de composition gazeuse toutes les 30 minutes), une moyenne sur deux jours a été utilisée afin de faciliter la lecture et de s'affranchir des variations journalières qui ne présentent pas d'intérêt pour cette étude.

Ces résultats, en concordance avec les nombreuses mesures ponctuelles effectuées lors de la phase de reconnaissance préalable du phénomène entre 1999 et 2000 (Pokryszka et Grabowski, 2002), ont montré clairement l'existence d'une corrélation entre le sens (et l'intensité) du flux d'air et la température extérieure. Aucun autre paramètre, influençant à long terme les écoulements (comme la pression atmosphérique par exemple), n'a pu être identifié.

Cette constatation nous a amenés à considérer le tirage naturel thermique comme le phénomène jouant un rôle primordial dans la formation des écoulements gazeux entre les réservoirs miniers et la surface. Ce phénomène est avant tout lié au différentiel de température existant entre l'atmosphère externe et les vieux travaux. Dans ces derniers, la température est habituellement quasiment constante (elle a été évaluée à 12 . $14^{\circ} \mathrm{C}$, dans notre cas), contrairement à la température atmosphérique, dont le niveau général varie selon le cycle saisonnier annuel.

De même, une configuration topographique de la surface au-dessus du réservoir minier de MoyeuvreGrande et d'autres réservoirs concernés semble favoriser le tirage thermique.

En effet, les secteurs étudiés se caractérisent globalement par une topographie très contrastée, avec des plateaux assez vastes, entrecoupés de manière franche par un réseau irrégulier de vallées. Le gisement ferrifère se situe à l'aplomb des plateaux, avec des affleurements apparaissant assez fréquemment sur les coteaux des vallées.

Durant la période estivale, une partie de l'air atmosphérique restant en contact avec les vieux travaux situés dans les zones surélevées (orifices mal ou non obturés, crevasses) est soumise à un refroidissement progressif. Une augmentation de la masse volumique, induite par l'effet du refroidissement, peut provoquer la migration de gaz vers les parties les plus basses des vieux travaux miniers non ennoyés. Le gaz sort ensuite du réservoir minier par les différentes ouvertures et orifices situés principalement le long des affleurements du gisement, dans les vallées. Une circulation d'air s'établit ainsi, dont le schéma de principe est montré à la figure 4. Le sens des écoulements y est représenté pour la période estivale.

Le fonctionnement du réservoir selon ce modèle se caractérise par deux périodes bien distinctes :

- une période de régime estival, pendant laquelle le flux reste globalement sortant du massif. Elle correspond à une température extérieure significativement supérieure à celle régnant dans le réservoir minier (environ $\left.14^{\circ} \mathrm{C}\right)$;

- une période de régime d'écoulement hivernal, pendant laquelle le flux reste globalement entrant vers les vieux travaux miniers. Ce régime s'installe d'une manière franche lorsque la température extérieure est inférieure à la température à l'intérieure des vieux travaux miniers (environ $12^{\circ} \mathrm{C}$ ).

Ces deux régimes sont séparés par des périodes transitoires, pendant lesquelles l'écoulement est fluctuant.

Une relation globale, présentée sur la figure 5, montrant le niveau des teneurs en $\mathrm{O}_{2}$ et $\mathrm{CO}_{2}$ mesurées pendant une longue période (plus d'un an), exprimé en fonction de la température atmosphérique, confirme la 
Température extérieure

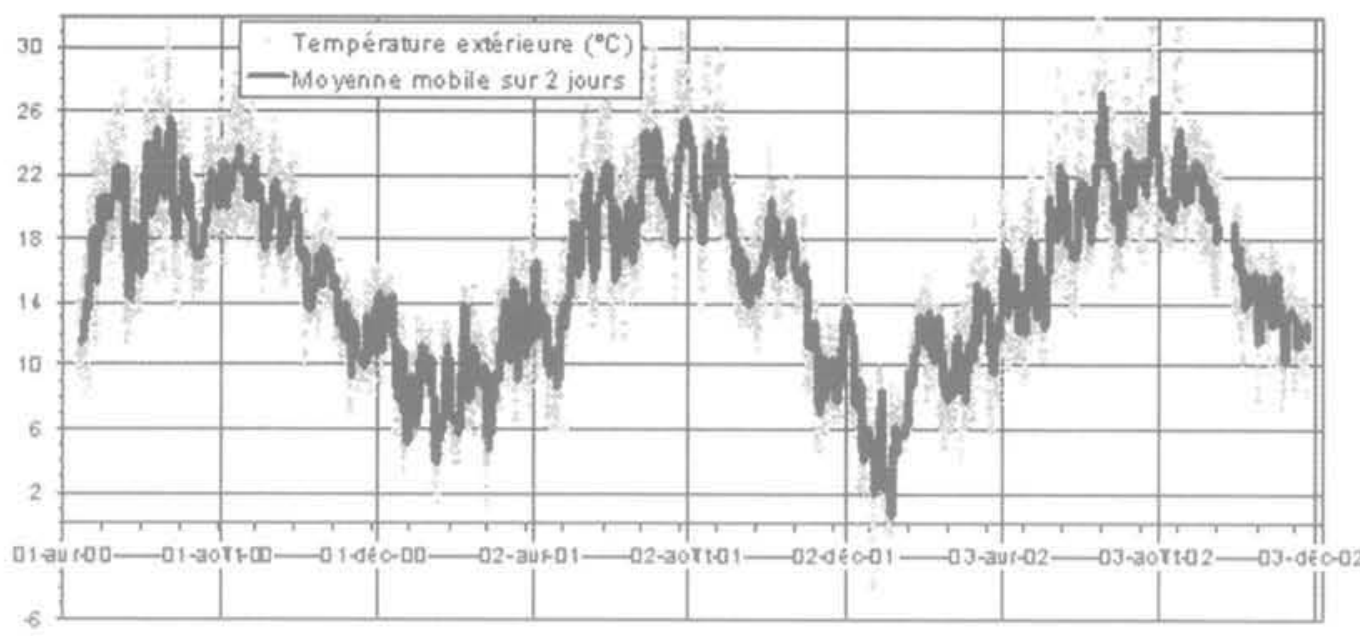

D ébit

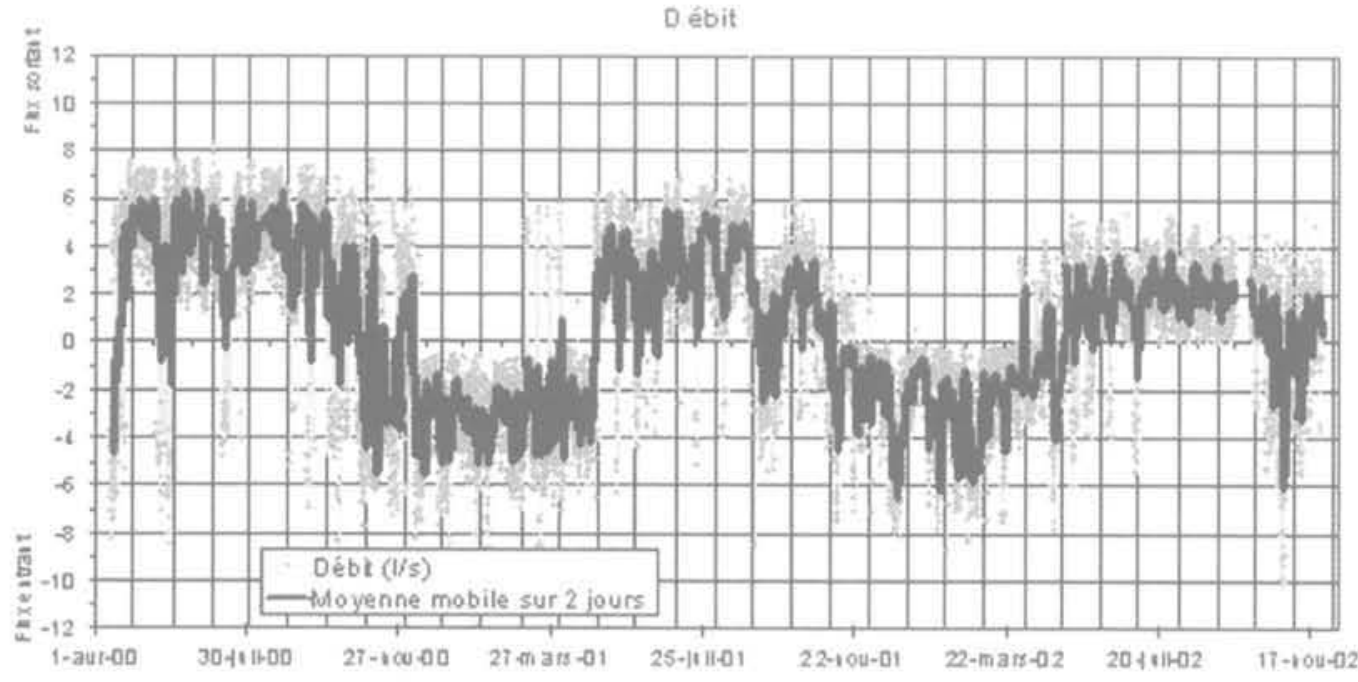

Radon

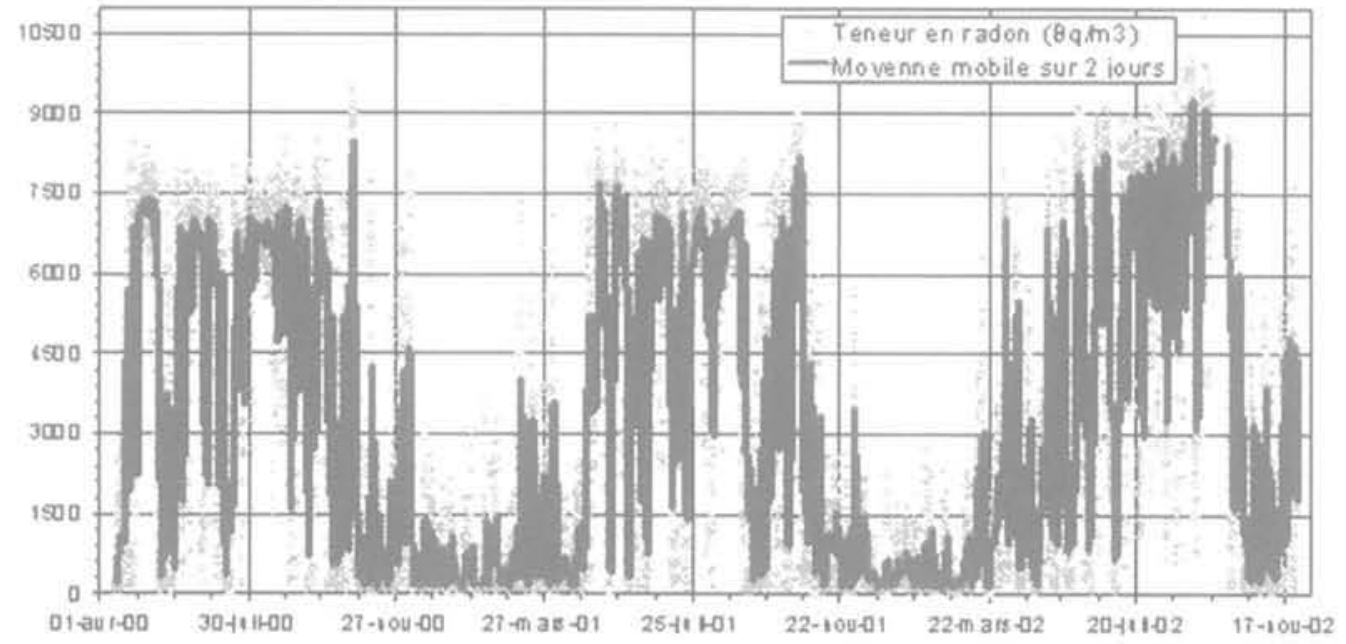

Fig. 2 Évolution de la température extérieure, du débit d'air et de la teneur en radon observés dans une galerie débouchant du réservoir de Moyeuvre-Grande.

Outside temperature, air flow rate and radon content evolutions observed in a gallery connected to the Moveuvre-Grande reservoir. 
Température extérieure

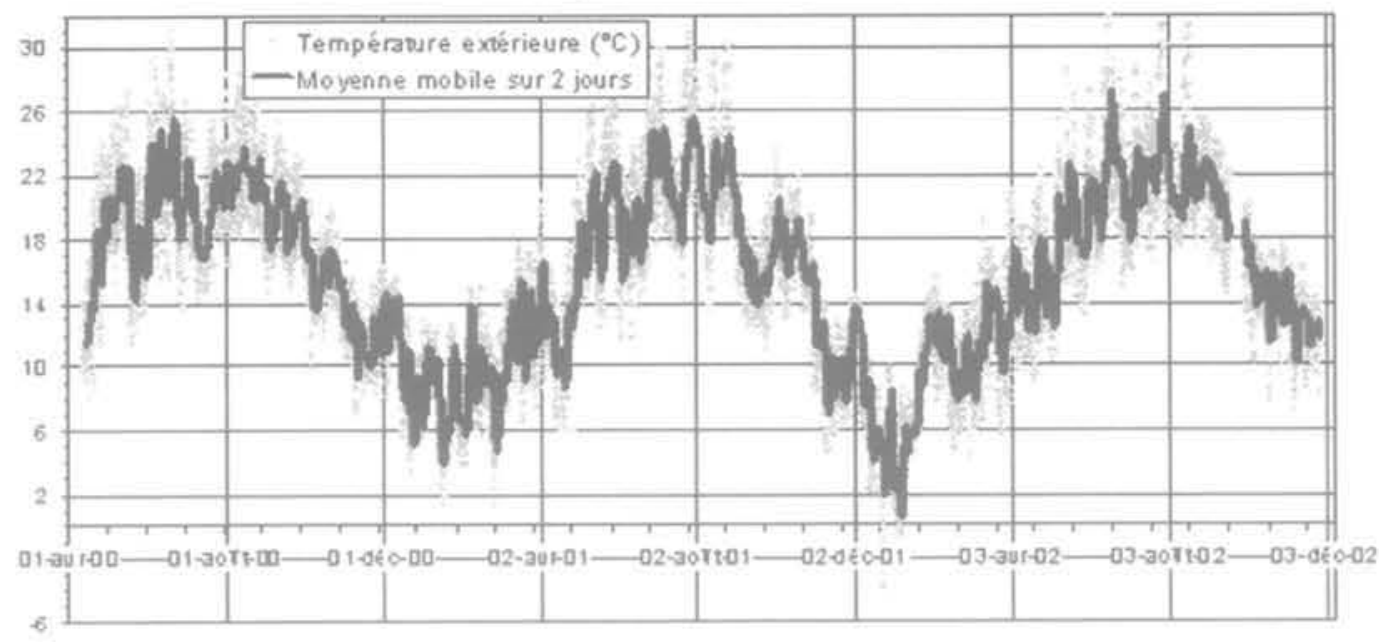

Teneur en oxygène

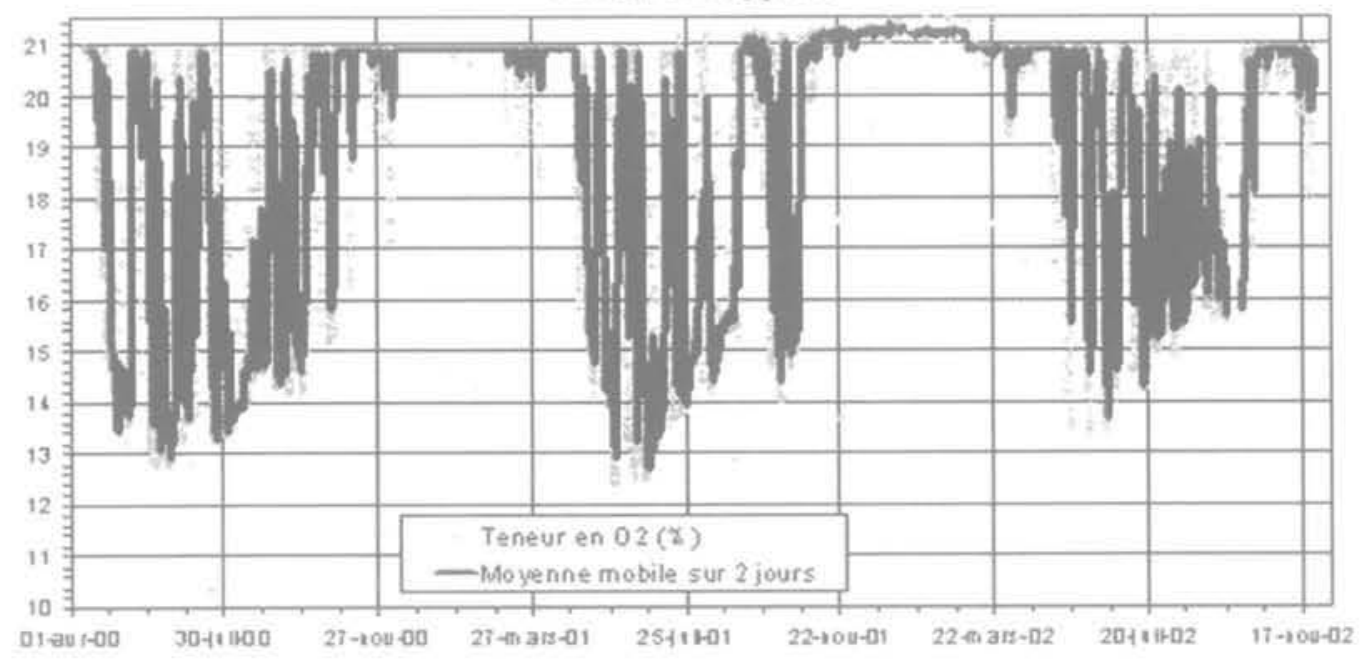

Teneur en dioxyde de carbone

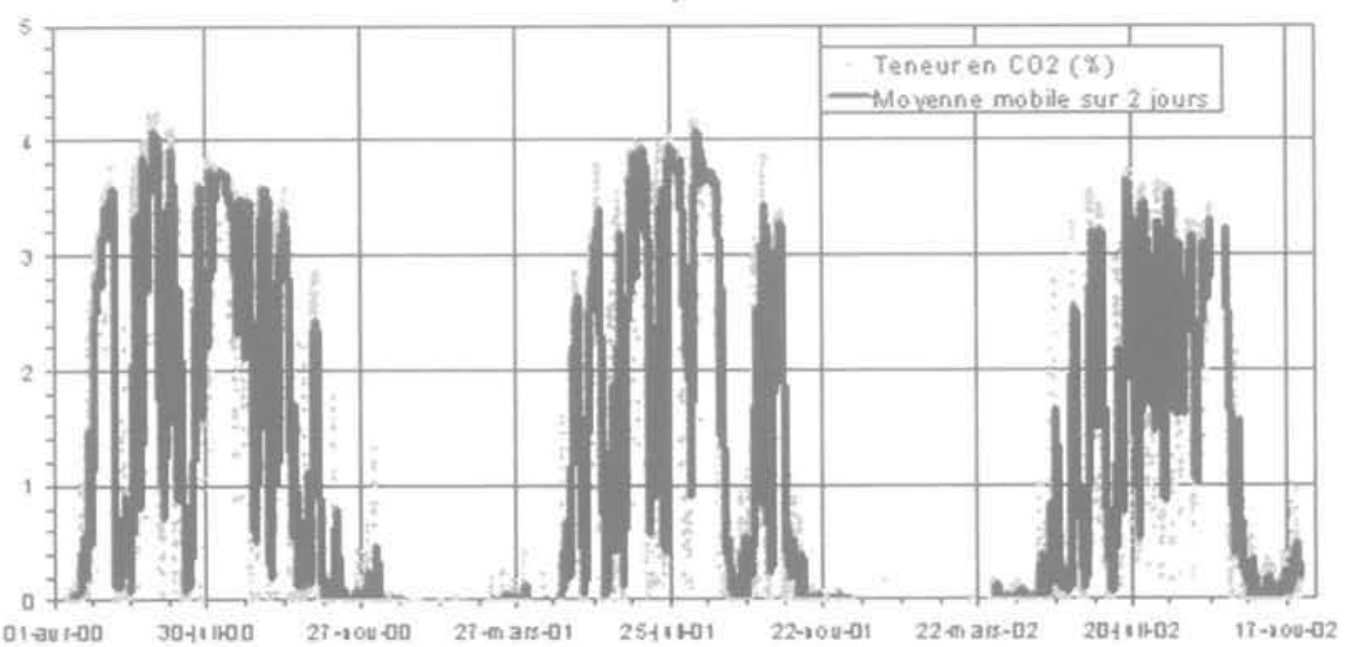

FG. 3 Évolution de la température extérieure et des teneurs en $\mathrm{CO}_{2}$, et $\mathrm{O}_{2}$ observées dans une galerie débouchant du réservoir de Moyeuvre-Grande.

Outside temperature, $\mathrm{CO}_{2}$ and $\mathrm{O}_{2}$ content evolutions observed in a gallery connected to the Moyeuvre-Grande reservoir. 


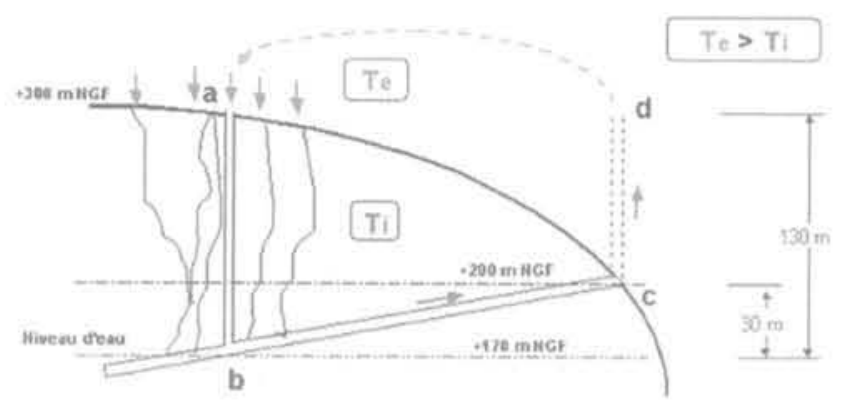

FIG.4 Schéma de principe du tírage naturel en période estivale (contexte du réservoir de Moyeuvre-Grande).

Schematic diagram of the natural thermal draught during the summer period (MoveuvreGrande reservoir case). pertinence du niveau limite de la température extérieure, identifié précédemment : l'atmosphère appauvrie en $\mathrm{O}_{2}$ et enrichie en $\mathrm{CO}_{2}$ apparaît pour des températures supérieures à $12-14^{\circ} \mathrm{C}$, ce qui correspond au régime de flux sortant du massif.

Par ailleurs, une simulation effectuée selon le modèle simplifié de Budryk (Budryk, 1929), a donné, pour les conditions du réservoir de Moyeuvre-Grande, une charge aéraulique due à ce phénomène de l'ordre de 20 à $30 \mathrm{~Pa}$, pour un différentiel de température de $10^{\circ} \mathrm{C}$. Cette charge semble largement suffisante pour mettre en place une circulation de gaz, dans le contexte donné.

Il est également à signaler que d'autres phénomènes naturels (variation de la pression barométrique, effet dynamique dû au vent, battement de la nappe aquifère...) s'ajoutent au phénomène du tirage nature! et peuvent perturber temporairement les deux régimes d'écoulement principaux caractérisés précédemment, sans pour autant pouvoir les inverser de manière durable.

Corrélation température extérieure - teneur en $\mathrm{CO} 2$

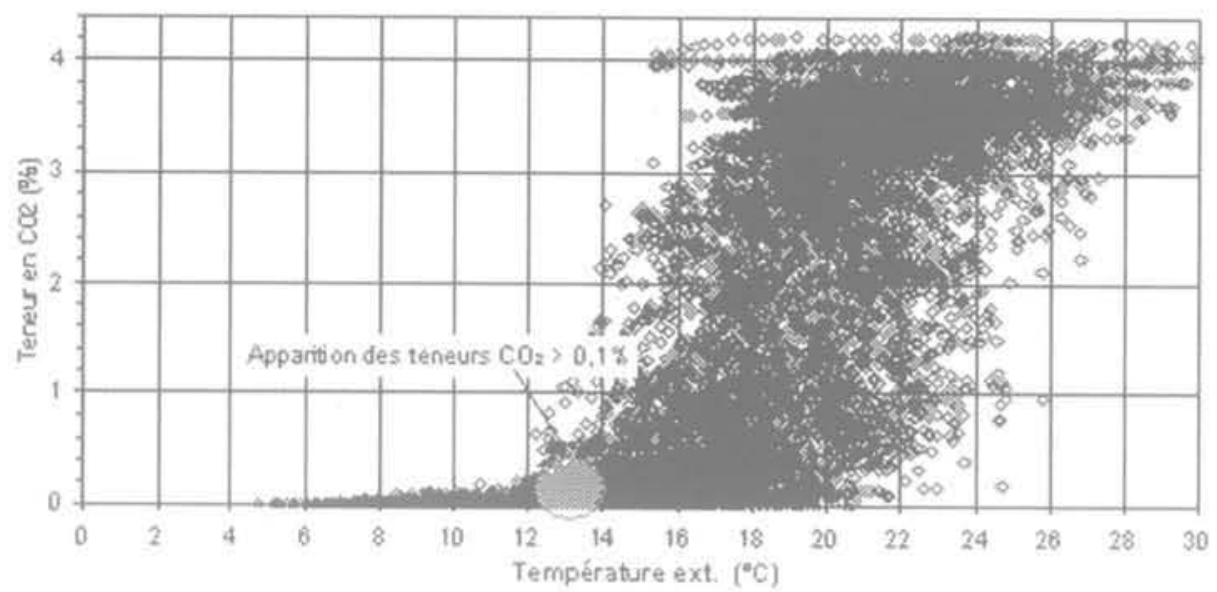

Corrélation température extérieure - teneur en oxygène

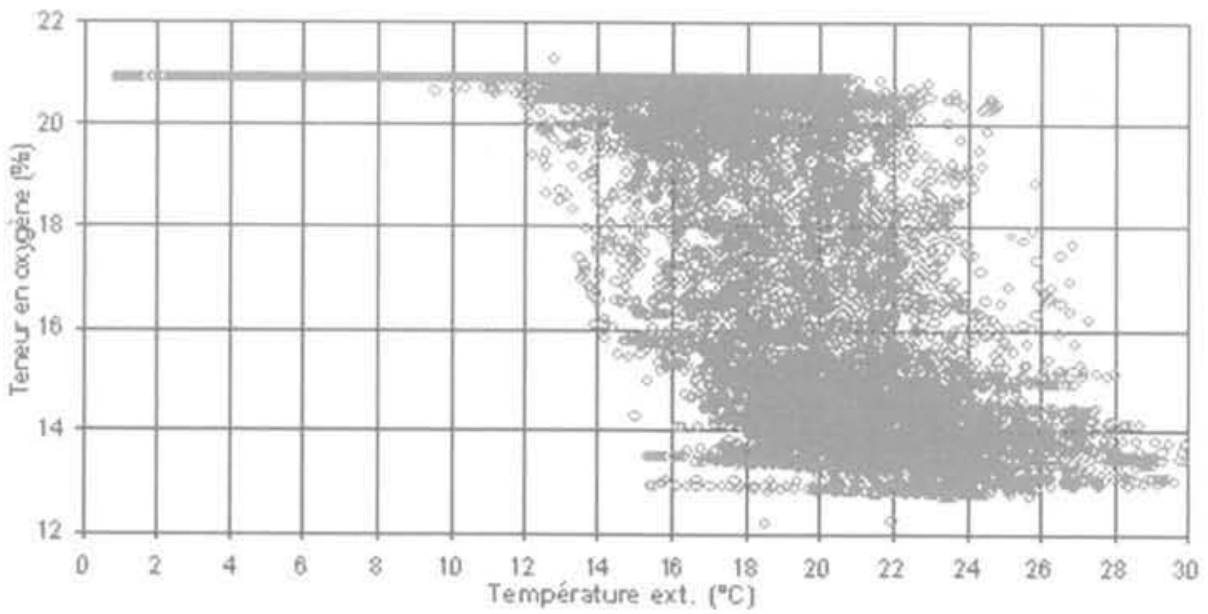

FIG.5 Corrélation entre la température extérieure et les teneurs en $\mathrm{O}^{2}$ et $\mathrm{CO}_{2}$ observées dans un orifice du réservoir de Moyeuvre-Grande.

Correlation between the outside temperature and the $\mathrm{O}_{2}$ and $\mathrm{CO}_{2}$ contents observed in an opening of the Moyeuvre-Grande reservoir. 


\section{Mécanisme de la modification de l'atmosphère dans les réservoirs miniers}

\section{1}

\section{Modification de l'atmosphère dans les réservoirs étudiés}

Les mesures de la composition de gaz émis à partir des vieux travaux miniers, effectuées en continu sur le réservoir de Moyeuvre-Grande et ponctuellement dans d'autres réservoirs de la partie nord du bassin ferrifère, ont montré que les modifications de l'atmosphère des vieux travaux miniers, constituée initialement d'air migrant plus ou moins librement par les terrains, consistent en une consommation de l'oxygène, accompagnée d'une génération moins que proportionnelle de dioxyde de carbone.

Cette information, recoupée avec les résultats d'une recherche bibliographique sur les réactions géochimiques observées dans les cas similaires d'exploitations minières (Feuga, 2000), a permis de retenir l'oxydation de la pyrite présente dans le gisement ferrifère, couplée à une dissolution de la calcite par l'acide sulfurique produit, comme l'hypothèse la plus probante pour expliquer le dégagement de $\mathrm{CO}_{2}$ et la consommation de $\mathrm{I}^{\prime} \mathrm{O}_{2}$,

Le bilan de cette réaction s'écrit :

$$
\begin{gathered}
\mathrm{FeS}_{2}+3,75 \mathrm{O}_{3}+2 \mathrm{CaCO}_{3}+1,5 \mathrm{H}_{2} \mathrm{O} \Leftrightarrow \mathrm{Fe}(\mathrm{OH})_{3} \\
+2 \mathrm{CO}_{2}+2 \mathrm{SO}_{4}^{2-}+2 \mathrm{Ca}^{2+}
\end{gathered}
$$

De plus, une étude menée par le Laboratoire environnement, géomécanique et ouvrages (LAEGO) a mis en évidence la présence de pyrite réactive dans les intercalaires marneux, riches en calcite, du gisement ferrifère lorrain (Collon et al., 2002),

Par ailleurs, une autre possibilité de vérifier l'hypothèse sur le mécanisme géochimique en question s'est présentée à l'occasion de l'expérimentation sur le site pilote de Tressange, consistant en un ennoyage contrôlé d'une petite partie de vieux travaux miniers.

\section{2}

\section{Modification de l'atmosphère dans le massif lors de son ennoyage (site pilote de Tressange)}

Afin d'étudier l'influence de l'ennoyage sur la stabilité des ouvrages miniers abandonnés et sur la qualité des eaux et la composition de l'atmosphère, une expérimentation spécifique a été entreprise dans le cadre du GISOS. Elle est réalisée dans les conditions réelles in situ sur le site pilote de Tressange.

Pour les besoins de cette expérimentation, une partie d'une ancienne mine exploitée par la méthode des chambres et piliers sur deux niveaux géologiques (couche brune et couche grise séparées par un intercalaire marneux) a été isolée, instrumentée et préparée afin d'effectuer son ennoyage contrôlé. Seule la couche la plus profonde (couche brune) ainsi que la partie inférieure de l'intercalaire marneux ont été ennoyées. Un schéma de principe du site expérimental est présenté sur la figure 6 .

L'INERIS a profité de cette opportunité pour essayer de vérifier l'influence de l'ennoyage du massif ferrifère sur la production éventuelle de gaz et sur la composition de l'atmosphère au sein du massif rocheux.

Ainsi, une expérimentation a été menée afin de suivre l'évolution de l'atmosphère dans l'enceinte isolée (teneurs en $\mathrm{O}_{2}$ et $\mathrm{CO}_{2}$ ), au cours de l'ennoyage. L'observation de l'atmosphère a été réalisée au moyen de deux forages traversant l'intercalaire marneux et débouchant au toit de l'enceinte, dans la couche brune (Fig. 6).

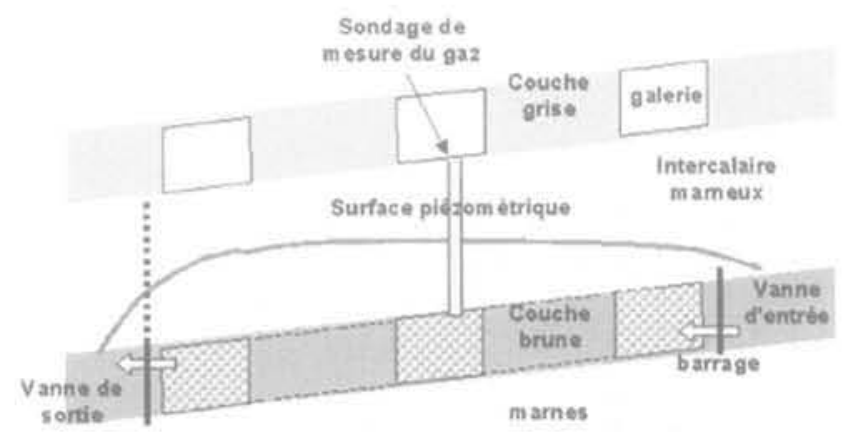

FIG. 6 Coupe schématique du dispositif de surveillance de l'atmosphère au cours de l'ennoyage du site pilote de Tressange.

Schematic section of the atmosphere watch over system during the flooding of the Tressange experimental site.

La figure 7 montre l'évolution des teneurs en $\mathrm{O}_{2}$ et $\mathrm{CO}$, mesurées dans les sondages au cours du temps, en parallèle avec la montée de l'eau d'ennoyage.

Ainsi, trois grandes phases ont pu être distinguées au cours de l'expérimentation :

- avant que l'eau n'atteigne le niveau de l'intercalaire marneux ( 35 premiers jours), la composition de l'atmosphère située à l'aplomb du sondage n'a pas évolué ; - lorsque le niveau de Y'eau a atteint la base de l'intercalaire marneux et a progressé au sein de cette couche (fin avril 2002), nous avons observé une évolution franche de la composition de l'air contenu dans le sondage qui s'appauvrit en $\mathrm{O}_{2}$ et s'enrichit en $\mathrm{CO}_{2}$;

- à partir du moment où le niveau d'eau s'est à peu près stabilisé dans l'intercalaire marneux, la composition de l'atmosphère du sondage n'a plus évolué.

Les résultats obtenus montrent ainsi clairement une liaison évidente entre la modification de l'atmosphère (consommation d'oxygène et production de dioxyde de carbone) sur le site expérimental et l'ennoyage de l'intercalaire marneux.

La modification de l'atmosphère serait donc consécutive à une réaction des marnes pyriteuses avec l'eau d'ennoyage, ce qui tend à confirmer notre hypothèse selon laquelle le dégagement de $\mathrm{CO}_{2}$ serait lié à une réaction associant la pyrite présente dans les marnes et la calcite dissoute par les acides produits. 
Teneurs en $\mathrm{O}_{2}$ et $\mathrm{CO}_{2}$

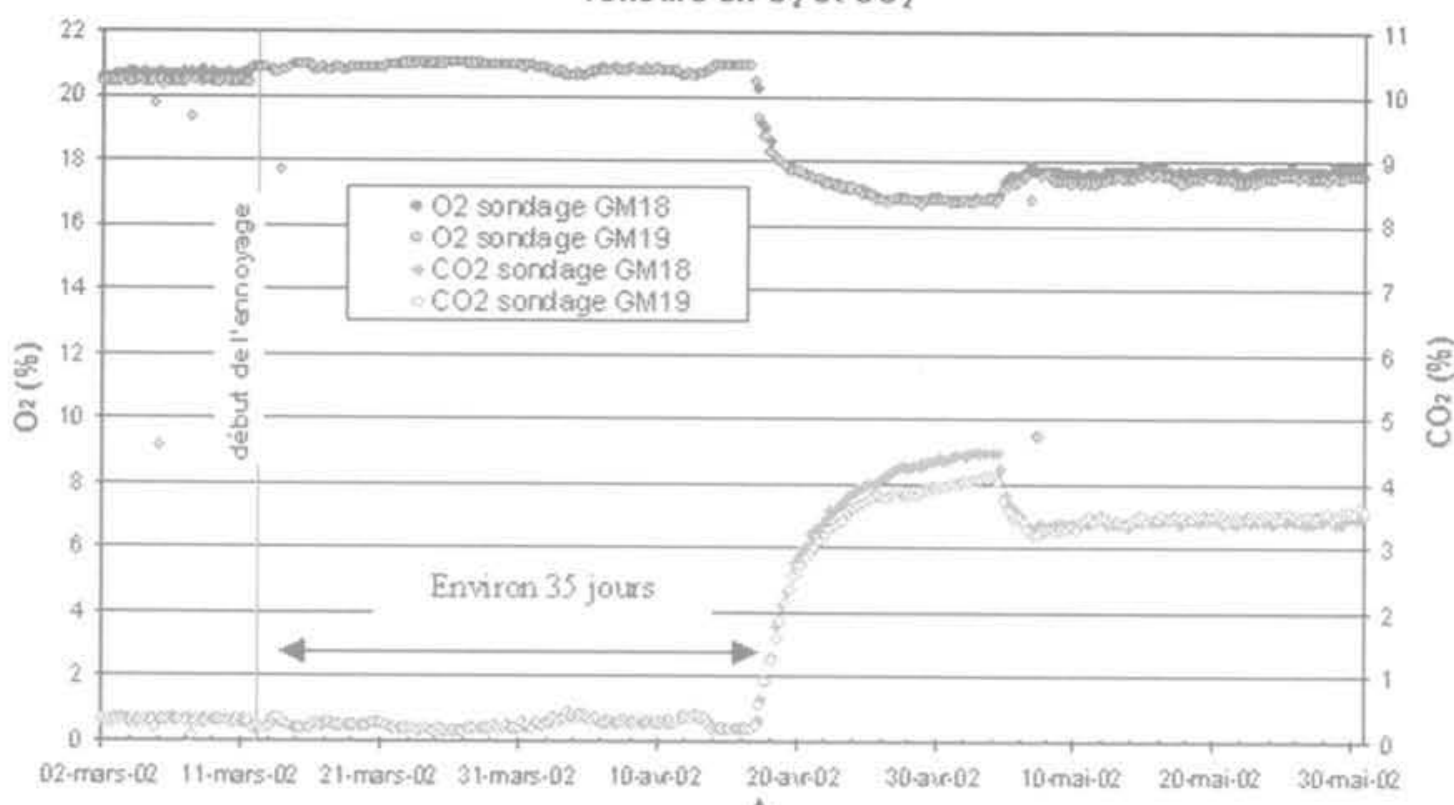

Niveau d'eau par rapport au toit de l'intercalaire marneux

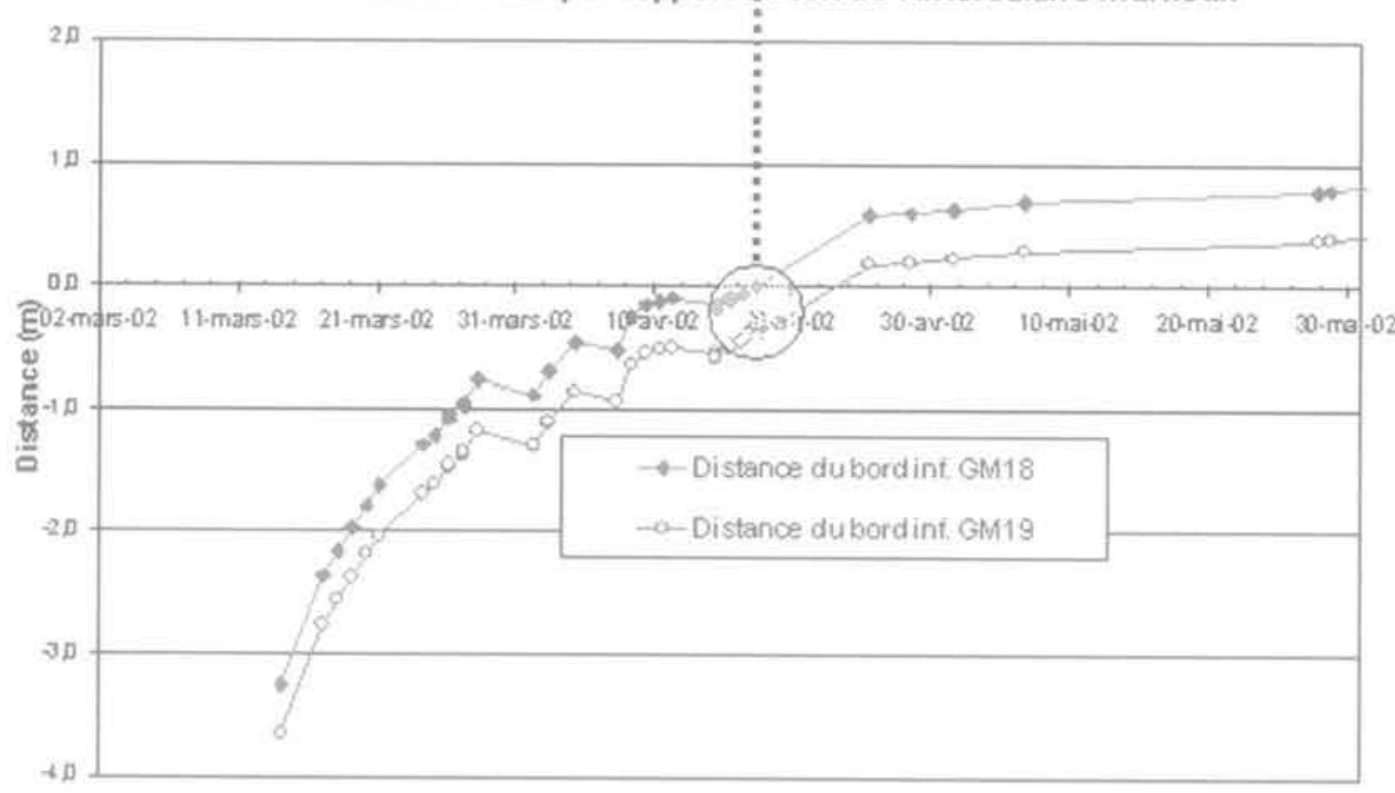

FIG,7 Évolution de la composition gazeuse dans le massif, au cours de l'ennoyage du site pilote de Tressange.

Evolution of the massif gaseous composition during the flooding of the Tressange pilot site.

Des analyses hydro-géochimiques menées en laboratoire sont en cours dans le cadre du programme GISOS, afin d'apporter des renseignements complémentaires sur la composition minéralogique du massif et de confirmer ce résultat obtenu in situ.

\section{5}

\section{Conclusion}

Les travaux miniers non ennovés ou partiellement ennoyés sont une source d'émission d'air vicié chargé en $\mathrm{CO}_{2}$ (et en radon). Les écoulements sont générés par le tirage naturel thermique et la modification de l'atmosphère initiale est vraisemblablement liée à une série de réactions chimiques impliquant la pyrite et la calcite de l'intercalaire marneux, au contact de l'eau et de l'air.

Cette dernière hypothèse semble confirmée par les études menées sur le site de Tressange où nous avons observé une forte évolution de la composition de l'atmosphère en contact avec l'intercalaire marneux.

L'ensemble de ces résultats laisse penser que l'ennoyage de mines actuellement hors d'eau peut entraîner une production de gaz $\left(\mathrm{CO}_{2} \ldots\right)$ et des émanations d'air vicié vers l'extérieur de la mine. 
Budryk W. - Depresja cieplna. Przeglad Gorniczo-Hutniczy, Nr 11, 12, 1929. Barrier P, Lorenz J. - Radon et santé. Géochronique $n^{\circ}$ 78, 2001.

Collon P. Fabriol R., Bues M. - Chemical changes in groundwater due to flooding of an iron mine in a non-acid-producing environment. Proceedings SWEMP 2002. p. $761-768$.
Feuga A. - Contribution à l'estimation des termes entrée sortie des gaz transitant dans d'anciens travaux miniers. Rapport de DESS CESTEMIN, Ecole des mines de Nancy, 2000.

Monomakhoff A. - Dangers résultant de la présence du grisou dans l'atmosphère de la mine. Rapport CERCHAR " GrisouGrisoumétrie-Anémométrie n, référencé CTO-Jli/MB 72-76-38, 1978.
Pokryszka Z, Grabowski D. - Rapport de synthèse sur lorigine et les mécanismes de circulation des gaz dans les vieux travaux du bassin ferrifere Lorrain. Rapport INERIS référencé DRS-02-25302/R04. 2002. 